\title{
Study of the Per OB2 star-forming complex
}

\section{The Compiled Catalogue of kinematic and photometric data}

\author{
A. N. Belikov ${ }^{1}$, N. V. Kharchenko², A. E. Piskunov ${ }^{1}$, E. Schilbach ${ }^{3}$, R.-D. Scholz ${ }^{4}$, and A. I. Yatsenko ${ }^{2}$ \\ 1 Intitute of Astronomy of the Russian Acad. Sci., 48 Pyatnitskaya Str., Moscow 109017, Russia \\ e-mail: abelikov@inasan.rssi.ru; piskunov@inasan.rssi.ru \\ 2 Main Astronomical Observatory, 27 Academica Zabolotnogo Str., 03680 Kiev, Ukraine \\ e-mail: nkhar@mao.kiev.ua; yatsenko@mao.kiev.ua \\ 3 Astronomisches Rechen-Institut, Mönchhofstraße 12-14, 69120 Heidelberg, Germany \\ 4 Astrophysikalisches Institut Potsdam, An der Sternwarte 16, 14482 Potsdam, Germany \\ e-mail: rdscholz@aip.de
}

Received 18 September 2001 / Accepted 6 December 2001

\begin{abstract}
In this paper we describe the construction of a Compiled Catalogue (CC) of about 30000 stars in a nearby region of star formation including the Per OB2 association. The CC is a photometric and astrometric survey with the center at $(\alpha, \delta)_{\mathrm{J} 2000}=\left(3^{\mathrm{h}} 51^{\mathrm{m}} 36^{\mathrm{s}}, 34^{\circ} 36^{\prime}\right)$ and radius of 10 degrees. The $\mathrm{CC}$ is based on recently published astronomical catalogues, e.g. Hipparcos and Tycho-2, and supplemented by relevant astrophysical data from numerous data sources. The CC is complete down to $V=11.6 \mathrm{mag}$, in general, and to $V=18.5 \mathrm{mag}$ in the one square degree field centered around the IC 348 cluster. We describe the reduction procedures allowing us to put the data onto one homogeneous system, i.e., the coordinates and proper motions on the Hipparcos system and the photometry on the Johnson system. Typical accuracies of $1 \ldots 20$ mas for coordinates, $1 \ldots 3$ mas/yr for proper motions and $0.01 \ldots 0.05 \mathrm{mag}$ for $B, V$ magnitudes were achieved for the bright $\mathrm{CC}$ stars $(V<12 \mathrm{mag})$. For $\approx 7000$ stars we also collected parallaxes and spectral classes. Down to the completeness limit of the CC, a preliminary proper motion selection yielded more than 1000 probable members of Per OB2. The catalogue is available in electronic form from the CDS at Strasbourg.
\end{abstract}

Key words. catalogs - stars: kinematics - stars: formation - open clusters and associations: individual: Per OB2

\section{Introduction}

Since early works by Ambartsumian (1947, 1949), stellar associations have been generally considered in the context of star formation. Due to the loose and irregular spatial structure of associations, kinematical data obtained in sky regions with associations provide important information to study their stellar content. In the pre-Hipparcos era, these studies were mainly restricted to the brightest stars in association areas (see e.g. Blaauw 1964). Consequently, there was room for speculation on subjects like the size of associations, their stellar content, and their kinematics.

With highly accurate all-sky data being available to the astronomical community since the completion of the Hipparcos-Tycho mission, one is able to study nearby

Send offprint requests to: E. Schilbach, e-mail: elena@ari.uni-heidelberg.de

* Catalogue is only available in electronic form at the CDS via anonymous ftp to cdsarc.u-strasbg.fr (130.79.128.5) or via

http://cdsweb.u-strasbg.fr/cgi-bin/qcat?J/A+A/384/145 associations for the total scope of problems using data on stars within a reasonable range of magnitudes and without any limitations on the sampling area. The first major result summarizing the Hipparcos programme observing nearby OB-associations is presented by de Zeeuw et al. (1999), who determined membership, average distances and kinematics of 12 stellar groups (including the Per OB2 association).

Our interest in the Per OB2 star-forming region arose from the study of a more compact region around the young cluster IC 348 (Scholz et al. 1999, hereafter S99). It was found that, apart from foreground field stars, the stellar population in the cluster area could be kinematically divided in two groups: one group was supposed to consist of cluster members, whereas the other was expected to represent the uniformly distributed distant field stars. It should be noted that, within the IC 348 area, Herbig (1998) also found evidence of the existence of a second population, which he attributed to the related population of the parent Per OB2 association. We decided to extend this study to the whole association in order to clarify this issue. 
As our previous experience has shown (Kharchenko \& Schilbach 1995; Belikov et al. 1999, 2000 for the NGC 6611 case), a wide field survey including accurate and complete spatio-kinematic, photometric and other relevant data (collected in a so-called compiled catalogue) is needed for a comprehensive study of complex embedded starforming structures like young clusters and embracing associations. These data are present in recent catalogues, such as Hipparcos (ESA 1997), Tycho-2 (Høg et al. 2000), Tycho-1 (ESA 1997), ACT RC (Urban et al. 1998b), TRC (Høg et al. 1998), CMC11 (CMC Nos. 1 to 11 1999), PPMN (Röser \& Bastian 1991), and FONAC (Kislyuk et al. 1999). The bulk of these catalogues is the outcome of the Hipparcos space mission, and they represent rather shallow surveys, since their completeness limit does not exceed 11.6 mag. Other catalogues could be added to increase the limiting magnitude of the survey. The IC 348 area, which is a region of special interest, is covered by the deeper S99 survey.

In the present study we were able to select more than 1000 probable association members out of almost 30000 stars collected in our Compiled Catalogue (CC hereafter). This should be compared with the previously known 17 classic members of Blaauw (1952), and 41 members from the Hipparcos catalogue alone (de Zeeuw et al. 1999).

This paper is the first in a series devoted to the comprehensive study of star formation in the Per OB2 association. It describes the observation data: the CC sources, reduction procedures, and the achieved accuracies. In the next paper we deal with morphology of the region and the stellar content of the association. Then we focus on stellar luminosity functions and color-magnitude diagrams (CMDs) in the whole region, and in IC 348 particularly.

This paper has the following structure. In Sect. 2 we describe the algorithm of the CC compilation. In Sect. 3 we consider principal data sources, reductions applied to various types of the data, and the resulting accuracies of the data. In Sect. 4 the kinematic selection method is briefly discussed and the results on membership determination are compared with recent results of de Zeeuw et al. (1999). Section 5 summarizes this work.

\section{The Compiled Catalogue construction}

Since there is a need for various types of data in a study of young stellar groups, it is worth compiling a catalogue from different sources, with sufficiently deep samples in at least the most important areas.

For this study we have used all published data in the sky area of the cluster IC 348 and the Per OB2 association.

Our stellar data sets are based on astrometric (coordinates and proper motions) and photometric data ( $B, V$ magnitudes transformed to the Johnson system). These values are determined for the bulk of selected stars, and they have been used for membership evaluation and a spatio-kinematic study of this region of star formation. Almost all brighter stars have HD and/or MK spectral
Table 1. Astrometric all-sky catalogues and the number of their stars included in the CC.

\begin{tabular}{lllr}
\hline & Catalogue & Reference & No of stars \\
\hline 1. & Hipparcos & ESA (1997) & 822 \\
2. & Tycho-2 & Høg et al. (2000) & 16032 \\
3. & Tycho-1 & ESA (1997) & 6876 \\
4. & ACT RC & Urban et al. (1998b) & 6374 \\
5. & TRC & Høg et al. (1998) & 6253 \\
6. & CMC11 & CMC Nos. 1 to 11 (1999) & 1350 \\
7. & PPM-N & Röser \& Bastian (1991) & 2439 \\
8. & FONAC & Kislyuk et al. (1999) & 25806 \\
\hline
\end{tabular}

classification. For about 6000 stars, trigonometric parallaxes are listed in the Hipparcos and Tycho surveys, but only $4 \%$ of them are accurate enough. 330 stars have measured radial velocities. In the small sky area with IC 348 we found additional data for faint stars: magnitudes in $U$, $R, H, I, J$ and $K$ bands, the results of X-ray and $\mathrm{H}_{\alpha}$ emission observations. We include these data in our catalogue.

The Compiled Catalogue contains two samples of stellar data. The deep sample $(V \lesssim 21)$ covers a relatively small rectangular area with the IC 348 cluster $\left(\mathrm{RA}, \mathrm{Dec}_{\mathrm{J} 2000}=3^{\mathrm{h}} .744,+32^{\circ} .15\right.$ degree$)$ and a size of 1 square degree, and the brighter and shallow one $(V<$ $12 \ldots 15)$ belongs to a larger circular area with a radius of 10 degrees centered at RA, Dec $\mathrm{J}_{2000}=3^{\mathrm{h}} 86,+34.6$ (assumed to be the Per OB2 core center). This is why the CC was constructed in several steps.

Step 1: In the first step we selected stars from eight astrometric all-sky catalogues in the large circular sky area. The list of the catalogues and the number of selected stars are given in Table 1. These catalogues represent the largest and most homogeneous set of proper motions and $B, V$ magnitudes for the whole sky. We cross-identified these catalogues by coordinates (proper motions, magnitudes, and BD numbers were used as a secondary criterion in doubtful cases, e.g. for optical pairs). After crossidentification our list contains 26551 stars.

Step 2: There is a number of studies of the Per OB2 association based on multi-color photoelectric photometry in the Vilnius and Johnson systems and MK classification. These lists are not complete, but they contain more accurate photometry for fainter stars compared to the above data set. We added data of Černis $(1990,1993)$, who published two dimensional photometric quantification and interstellar absorption on the basis of the Vilnius photometric system for about 270 stars in two fields of the Perseus complex. We have also used a number of smaller lists: Guetter (1977), Seyfert et al. (1960) and Harris (1956), giving the results of original photometry in the Johnson optical and near-infrared systems and MK classification for more than 200 stars distributed over the Per OB2 association area. Since these lists do not contain coordinates, we identified these stars by use of $\mathrm{BD} / \mathrm{HD}$ numbers only. Further, we selected 6784 stars with MK/HD spectral classes from the SIMBAD database. Radial velocities and X-ray data available in SIMBAD for this region were 
Table 2. Stellar data used in the IC 348 field.

\begin{tabular}{|c|c|c|c|c|}
\hline $\begin{array}{l}\text { Reference to } \\
\text { a catalogue }\end{array}$ & $\begin{array}{l}\text { No of } \mu U B V R I \\
\text { stars }\end{array}$ & $J H K \mathrm{X}$ & $\mathrm{KS}$ & \\
\hline S99 & $1679+++++$ & & & \\
\hline Fredrick (1956) & $38+\quad++$ & & & \\
\hline Herbig (1998) & ++++ & & + & + \\
\hline Trullols \& Jordi (1997) & +++++ & & & \\
\hline Lada \& Lada (1995) & 510 & +++ & & \\
\hline Luhman et al. (1998) & 140 & & + & + \\
\hline Strom et al. (1974) & +++ & + & + & \\
\hline Preibisch et al. (1996) & 116 & + & & \\
\hline
\end{tabular}

also included in the CC. After this step our catalogue contains 27508 objects.

Step 3: We selected data for fainter stars from catalogues in the small sky area with the cluster IC 348. This step is independent of previous ones.

First, we used recent data by S99 who obtained a new proper motion survey for more than 1500 stars with $R<18 \mathrm{mag}$ in a one square degree region containing IC 348. This survey gives the deepest and the largest proper motion sample for this sky region. Note that before only relative proper motions for 38 stars down to $13.5 \mathrm{mag}$ of Fredrick (1956) were available in the IC 348 field.

We also used a number of recent catalogues with different astrophysical data for stars in the central area of IC 348. These catalogues, numbers of selected stars and data types are listed in Table 2. Data on interstellar absorption from Lada \& Lada (1995) and Strom et al. (1974) and X-ray luminosities from Preibisch et al. (1996) were also included in the CC. The resulting deep part of the CC survey contains 2158 stars.

Step 4: We merged the shallow and deep surveys with this step. The final compiled catalogue contains 29452 stars. Table 3 gives the overview on data collected in the $\mathrm{CC}$.

\section{The data}

The bulk of the CC stars were selected from eight catalogues listed in Table 1.

The Hipparcos and Tycho-1 catalogues are the most important sources of accurate and homogeneous information for studies based on astrometric and photometric data. Though Tycho-1 provides only very poor accuracies of proper motions and parallaxes for faint stars, at $V<8$ the Tycho-1 precision is acceptable for the goals of this study.

The catalogues ACT RC and TRC have been constructed on the basis of two all-sky catalogues: the Tycho-1 catalogue and the Astrographic Catalogue (AC). Due to the large epoch difference between the Tycho and AC catalogues, both ACT RC and TRC provide proper motions of high accuracy (standard errors better than 2.5 mas/yr). ACT RC and TRC have almost identical stellar content and differ due to the identification techniques applied to Tycho/AC, and due to the reduction models accepted
Table 3. Contents of the Compiled Catalogue.

\begin{tabular}{|c|c|c|}
\hline Data & $\begin{array}{l}\text { Star Area } \\
\text { No. }\end{array}$ & Notes \\
\hline $\mathrm{RA}_{\mathrm{J} 2000}$ & 29452 large & $3.048 \ldots 4^{\mathrm{h}} 675$ \\
\hline Dec J2000 & 29452 large & $+24^{\circ} .50 \ldots+44^{\circ} .57$ \\
\hline Hipparcos identification & 852 large & \\
\hline HD identification & 2880 large & \\
\hline BD identification & 2687 large & \\
\hline Variability flags & 903 large & $\begin{array}{l}\text { from Tycho-1, Hip } \\
\text { CMC11 }\end{array}$ \\
\hline Duplicity flags & 6873 large & $\begin{array}{l}\text { from Tycho-1 -2, Hip } \\
\text { CMC11, PPM-N }\end{array}$ \\
\hline Trig. parallax, $\pi, \varepsilon_{\pi}$ & 6751 large & $\begin{array}{l}738 \text { stars with } \\
\varepsilon_{\pi}<6 \text { mas, } \pi>\varepsilon_{\pi}\end{array}$ \\
\hline Proper motions, $\mu, \varepsilon_{\mu}$ & 28321 large & $\begin{array}{l}21529 \text { stars with } \\
\varepsilon_{\mu}<5 \mathrm{mas} / \mathrm{yr}\end{array}$ \\
\hline$B$ magnitude, $\varepsilon_{B}$ & 28560 large & $\begin{array}{l}2.77 \ldots 22.57 \mathrm{mag} \\
\varepsilon_{B}<0.05 \mathrm{mag} \\
\text { for } 2130 \mathrm{stars}\end{array}$ \\
\hline$V$ magnitude, $\varepsilon_{V}$ & 26727 large & $\begin{array}{l}2.88 \ldots 21.22 \mathrm{mag} \\
\varepsilon_{V}<0.05 \mathrm{mag} \\
\text { for } 2920 \mathrm{stars}\end{array}$ \\
\hline$U$ magnitude & 712 large & $1.72 \ldots 21.98 \mathrm{mag}$ \\
\hline$R$ magnitude & 1907 small & $8.07 \ldots 21.92 \mathrm{mag}$ \\
\hline$I$ magnitude & 302 small & $6.66 \ldots 20.59 \mathrm{mag}$ \\
\hline$J$ magnitude & 523 small & $6.28 \ldots 16.88 \mathrm{mag}$ \\
\hline$H$ magnitude & 523 small & $6.20 \ldots 16.23 \mathrm{mag}$ \\
\hline$K$ magnitude & 529 small & $3.76 \ldots 15.22 \mathrm{mag}$ \\
\hline Color-excess, $E_{(B-V)}$ & 469 large & $0.00 \ldots 1.68 \mathrm{mag}$ \\
\hline Absorption $A_{V}$ & 296 large & $0.00 \ldots 8.27 \mathrm{mag}$ \\
\hline Absorption $A_{J}$ & 121 small & $0.00 \ldots 4.93 \mathrm{mag}$ \\
\hline X-ray count, $\varepsilon_{\text {count }}$ & 114 small & $0.4 \ldots 43.1 \mathrm{cnts} / \mathrm{ksec}$ \\
\hline$W\left(\mathrm{H}_{\alpha}\right)$ & 124 small & $0 \ldots 235 \AA$ \\
\hline Spectral class & 7018 large & \\
\hline Radial velocity, $V_{\mathrm{R}}$ & 330 large & $-199 \ldots+223 \mathrm{kms}^{-1}$ \\
\hline
\end{tabular}

for the AC. That leads to random differences between ACT RC and TRC proper motions of about 2.65 mas/yr (Høg et al. 1998).

The most recent catalogue of the Hipparcos-Tycho set is the Tycho- 2 catalogue with positions, proper motions and $B_{\mathrm{T}}, V_{\mathrm{T}}$ magnitudes for 2.5 million stars (Høg et al. 2000). The positions and magnitudes were determined from a re-reduction of the Tycho data. The proper motions were derived using a new reduction of 144 ground-based astrometric catalogues, including the AC. Due to the large time base-line, the Tycho-2 proper motions are as accurate as the Hipparcos proper motions. The systematic errors of Tycho- 2 proper motions are less than about $0.5 \mathrm{mas} / \mathrm{yr}$ on angular scales of more than $6^{\circ}$ (Høg et al. 2000). The observed Tycho-2 positions have no significant differences with respect to the Hipparcos ones. The Tycho-2 survey is about 1 mag deeper than Tycho- 1 .

The FONAC catalogue is the result of a photographic survey of the northern sky carried out during the 1990 s. The positions and $B$ magnitudes of 2 million stars were determined on the basis of measurements of plates obtained with the wide-angle astrograph of the Main Astronomical 
Observatory in Kiev with respect to the ACT RC stars. The AC2000 (Urban et al. 1998a) is used as the first epoch for the determination of proper motions. Additionally, $V$ magnitudes were taken from the Tycho-1 and GSC 1.1 catalogues. The rms errors of the coordinates, proper motions and stellar magnitudes are $0.2 \operatorname{arcsec}, 3 \mathrm{mas} / \mathrm{yr}$ and $0.2 \mathrm{mag}$, respectively. Since the AC was used as the input catalogue of FONAC, the limiting magnitude is different for different sky areas.

The catalogue CMC11 is a compilation of the Carlsberg Meridian Catalogues (1999), observed with the Automatic Meridian Circle on La Palma between May 1984 and May 1998. The catalogue mainly comprises positions, proper motions and $V$ magnitudes for stars north of declination $-40^{\circ}$ of different astrometric and stellar-statistics programmes, including about 6000 stars in nearby O-B associations. In our region it contains stars fainter than the limiting magnitudes of most of the other catalogues listed in Table 1 . The positions of stars in CMC11 were combined with PPM and AC positions at previous epochs in order to derive their proper motions in the ICRS with standard errors less than 3... 4 mas/yr.

The PPM-N catalogue is the product of the compilation of all positional observations on the Northern sky made before the early $60 \mathrm{~s}$. Positions of stars in different catalogues including AC were used for PPM-N construction and proper motion calculations. The astrometric system of PPM-N is that of FK5, being close to ICRS. The standard errors of proper motions in PPM-N are better than 5 mas/yr (Kharchenko et al. 1995).

The main reason for using the PPM-N as well as CMC11 was that proper motions for some stars in this region were only found in these catalogues.

\subsection{Equatorial coordinates}

The cross-identifications were performed on the basis of equatorial coordinates, being transformed to equinox J2000.0 if necessary, and to the Hipparcos epoch, if the proper motions were available. Some catalogues do not contain accurate positions. A few catalogues (Seyfert et al. 1960; Guetter 1977; Harris 1956; Strom et al. 1974) do not provide any coordinates; these stars were identified by $\mathrm{BD} / \mathrm{HD}$ or other relevant identifiers given in these papers.

Table 4 gives the matching radii used in the identification in different catalogues deduced from comparison of coordinates and reflecting the specific positional accuracy in these catalogues. The catalogues are listed in Table 4 according to the priority used for the CC equatorial coordinates. The coordinates are given for equinox J2000 and Hipparcos epoch 1991.25 (if proper motions were available).

\subsection{Proper motions}

Since our shallow survey contains a sufficient number of Hipparcos stars, it is possible to transform proper motions
Table 4. Identification radius in various catalogues.

\begin{tabular}{lr}
\hline Catalogue & $r$, arcsec \\
\hline Hipparcos & 1 \\
Tycho-2 & 1 \\
Tycho-1 & 1 \\
CMC11 & 1.5 \\
PPM-N & 4 \\
FONAC & 4 \\
S99 & 5 \\
Fredrick (1956) & 7 \\
Herbig (1998) & 8 \\
Trullols \& Jordi (1997) & 10 \\
Cernis (1990, 1993) & 20 \\
Lada \& Lada (1995) & 10 \\
Strom et al. (1974) & 10 \\
Preibisch et al. (1996) & 10 \\
SIMBAD & $3^{\mathrm{a}}$ \\
\hline a For some objects 15 arcsec.
\end{tabular}

${ }^{\mathrm{a}}$ For some objects 15 arcsec.

from other catalogues to the Hipparcos system, which presently defines the celestial reference system.

Strictly speaking, only Hipparcos and Tycho-1 in Table 1 can be regarded as independent catalogues of proper motions in comparison with other catalogues used for the $\mathrm{CC}$ compilation. Over its whole magnitude range, Hipparcos contains highly accurate proper motions, both systematically and randomly.

Proper motions of the above eight catalogues were transformed to the Hipparcos system with equations taking into account the zero-point and magnitude-dependent terms:

$\mu_{X}^{\text {Hip }}-\mu_{X}^{\text {catalogue }}=a_{X} V+b_{X}$,
$\mu_{Y}^{\text {Hip }}-\mu_{Y}^{\text {catalogue }}=a_{Y} V+b_{Y}$.

Directions of axes $X$ and $Y$ coincide with those of right ascension and declination. The zero-point term contains all regional corrections including corrections for the precession constants.

The systems of these equations were solved for common stars by the least-squares method with the rejection of stars not passing a 3-sigma criterion. The common stars in Hipparcos and a corresponding catalogue were selected by the condition that the differences of their proper motions and the rms errors were less than 5 mas/yr. Table 5 gives the results of these solutions.

We used these results to transform the proper motions of the astrometric catalogues to the Hipparcos system. The proper motions and their errors in the shallow survey were computed as the weighted mean. The weights were determined in accordance with rms errors of individual stars in each catalogue used. The proper motion differences between the $\mathrm{CC}$ and other catalogue data are shown in Fig. 1.

A deep sample of proper motions given in S99 was constructed on the basis of a survey in a one square degree field centered at IC 348. Data from POSS Schmidt plates 
Table 5. Results of proper motion comparison of different catalogues with Hipparcos.

\begin{tabular}{lccccccc}
\hline Catalogue & $\begin{array}{c}\text { Number } \\
\text { of stars }\end{array}$ & $\begin{array}{c}\text { rms error of } \mu_{X} \\
{[\mathrm{mas} / \mathrm{yr}]}\end{array}$ & $\begin{array}{c}a_{X} \\
{[\mathrm{mas} / \mathrm{yr} / \mathrm{mag}]}\end{array}$ & $\begin{array}{c}b_{X} \\
{[\mathrm{mas} / \mathrm{yr}]}\end{array}$ & $\begin{array}{c}\text { rms error of } \mu_{Y} \\
{[\mathrm{mas} / \mathrm{yr}]}\end{array}$ & $\begin{array}{c}a_{Y} \\
{[\mathrm{mas} / \mathrm{yr} / \mathrm{mag}]}\end{array}$ & $\begin{array}{c}b_{Y} \\
{[\mathrm{mas} / \mathrm{yr}]}\end{array}$ \\
\hline Tycho-2 & 750 & 1.82 & $+0.02 \pm 0.05$ & $-0.14 \pm 0.45$ & 1.64 & $+0.05 \pm 0.05$ & $-0.36 \pm 0.41$ \\
Tycho-1 & 201 & 4.23 & $+0.24 \pm 0.30$ & $-1.69 \pm 2.08$ & 3.51 & $-0.08 \pm 0.25$ & $+0.34 \pm 1.71$ \\
ACT RC & 634 & 2.94 & $+0.14 \pm 0.10$ & $-1.35 \pm 0.81$ & 2.62 & $+0.07 \pm 0.09$ & $-0.59 \pm 0.73$ \\
TRC & 685 & 2.46 & $+0.11 \pm 0.08$ & $-0.85 \pm 0.71$ & 2.46 & $+0.24 \pm 0.09$ & $-2.14 \pm 0.73$ \\
FONAC & 678 & 3.57 & $+0.26 \pm 0.12$ & $-2.19 \pm 1.01$ & 3.53 & $+0.07 \pm 0.12$ & $-0.64 \pm 1.00$ \\
CMC11 & 511 & 3.27 & $+0.33 \pm 0.13$ & $-3.72 \pm 1.07$ & 3.37 & $+0.09 \pm 0.13$ & $+0.62 \pm 1.10$ \\
PPM-N & 570 & 4.00 & $+0.17 \pm 0.15$ & $-1.49 \pm 1.26$ & 3.99 & $+0.22 \pm 0.15$ & $-1.76 \pm 1.25$ \\
\hline
\end{tabular}

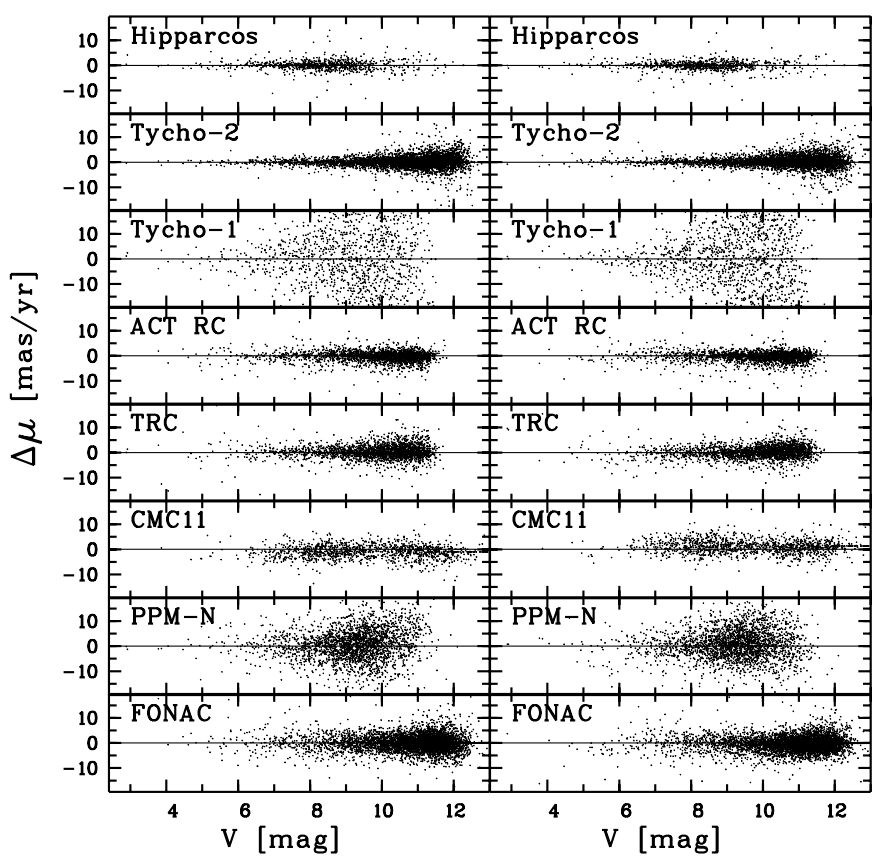

Fig. 1. The proper motion differences in $X$ (left panels) and in $Y$ (right panels) between the $\mathrm{CC}$ and individual catalogues versus $V$-magnitude.

(scale $67.2 \mathrm{arcsec} / \mathrm{mm}$ ) were involved. The five POSS plates used for proper motion determination are covering a time baseline of about 37 years.

Unfortunately, there are only a few common stars in S99 and in the shallow survey. Therefore, we are not able to transform S99 proper motions directly to the Hipparcos system. To improve the situation, we need to compare these catalogues with intermediate data, including both bright and faint stars. Presently, only the catalogue of Fredrick (1956) can be used for this purpose. Fredrick's data show a very good (better than 0.5 mas/yr) random accuracy. They were determined with 56 plates obtained with the Sproul 24-inch refractor (scale of $18.87 \mathrm{arcsec} / \mathrm{mm}$ ) and the McCormick 26-inch refractor (scale of $20.57 \mathrm{arcsec} / \mathrm{mm}$ ) with a time baseline of about 40 years. Up to very recently, this was the only proper motion survey in the IC 348 field and has been widely used for the selection of cluster members. Our study has shown that the proper motions of Fredrick (1956) suffer from a large magnitude equation, especially in $\mu_{Y}$. As one can see
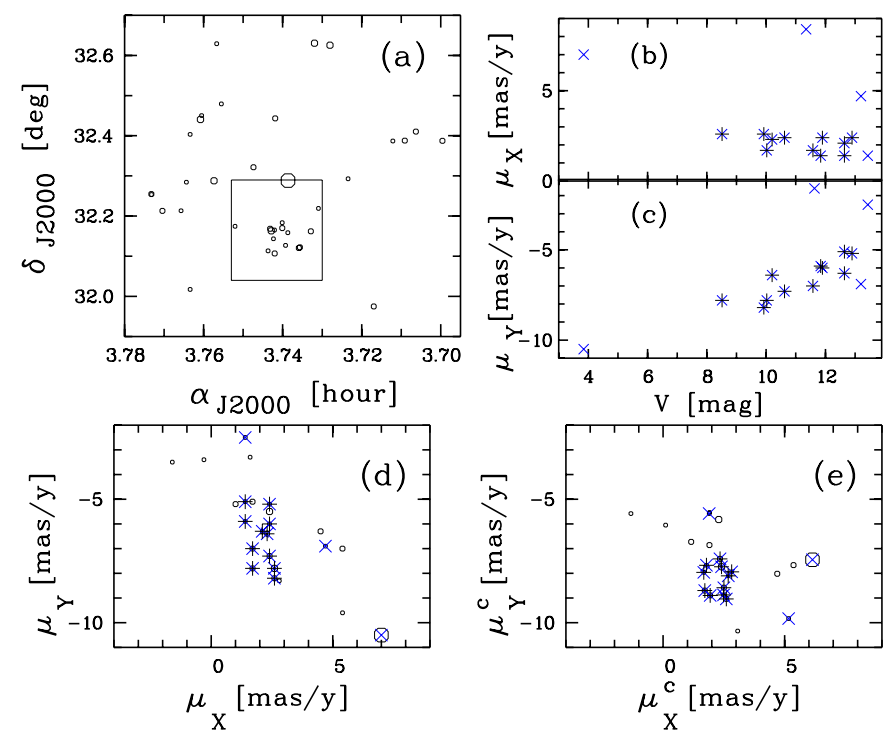

Fig. 2. Correction of proper motions of Frederick (1956) for the magnitude equation. Panel a) a map of the sky area with Fredrick's stars. The size of circles in panels a), d), e) corresponds to stellar brightness. A solid box indicates the central part of IC 348, where the majority of stars are cluster members. In panels $\mathbf{b}-\mathbf{e}$ ) stars from this box are marked with crosses, the cluster members according to proper motion selection are marked with asterisks. The original proper motions in $X$ and in $Y$ versus $V$ magnitude are shown in panels b) and c). The vector proper motion diagram with the original and corrected data are shown in panels $\mathbf{d}$ ) and $\mathbf{e}$ ), respectively.

in Fig. 2, proper motions of stars which could be regarded as cluster members from both spatial and kinematic criteria depend on the stellar magnitude. The corresponding magnitude equations are +0.141 and $-0.639 \mathrm{mas} / \mathrm{yr} / \mathrm{mag}$ in $\mu_{X}$ and $\mu_{Y}$, respectively.

After correction of Fredrick's proper motions for the magnitude equation we transformed these corrected data and the proper motions of S99 to a common system using 11 stars. The result was an "intermediate" catalogue composed of S99 and Frederick's data. There are 32 common stars in this intermediate catalogue and in the shallow survey that represent the Hipparcos system. The comparison of the results is shown in Fig. 3. 

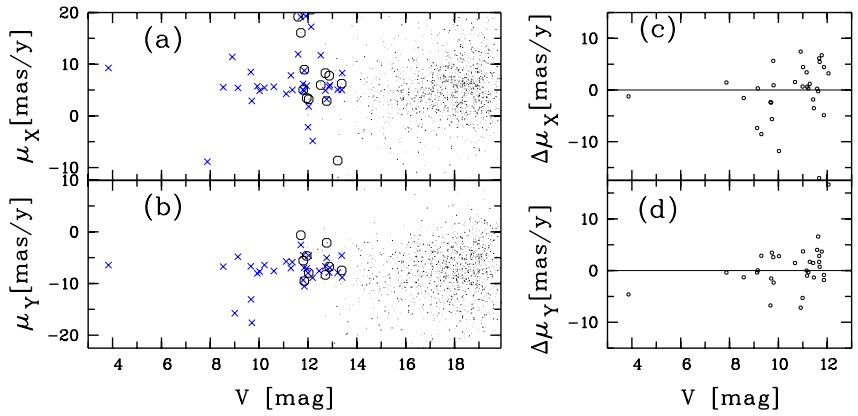

Fig. 3. The comparison of proper motions of faint and bright stars. Panels a) and b) crosses mark Fredrick's data, corrected for magnitude equation; dots and open circles correspond to S99 data, not common and common with Fredrick's stars, respectively. Panels c) and $\mathbf{d}$ ) proper motion differences between the shallow survey (in Hipparcos system) and the intermediate catalogue (Fredrick $1956+$ S99).

We found that the Fredrick (1956) and S99 proper motions can be reduced to the Hipparcos system by the following equations (in mas/yr):

$$
\begin{aligned}
\mu_{X}^{\mathrm{c}}-\mu_{X}^{\mathrm{Fr}} & =(+3.5 \pm 1.0)+(0.14 \pm 0.04)(V-12.4), \\
\mu_{Y}^{\mathrm{c}}-\mu_{Y}^{\mathrm{Fr}} & =(-1.4 \pm 0.8)-(0.64 \pm 0.05)(V-12.4), \\
\mu_{X}^{\mathrm{c}}-\mu_{X}^{\mathrm{S} 99} & =(+3.4 \pm 0.8), \\
\mu_{Y}^{\mathrm{c}}-\mu_{Y}^{\mathrm{S} 99} & =(-1.1 \pm 0.7) .
\end{aligned}
$$

As a result, the CC contains weighted mean proper motions in the Hipparcos system for 28321 stars, where 21529 and 3530 stars, respectively, have rms errors better than 5 and 2 mas/yr.

\subsection{Trigonometric parallaxes}

There are two sets of trigonometric parallaxes in the CC: the Hipparcos parallaxes with the presently highest accuracy (with rms errors of about 1 mas) and the Tycho-1 parallaxes with typically one order of magnitude lower accuracy for faint stars. We did not find any systematic differences between these data sets. Therefore, we calculated the trigonometric parallaxes as weighted means, with weights which are inversely proportional to squared rms errors. The CC contains trigonometric parallaxes in the Hipparcos system for 6751 stars, but only 3966 stars have positive parallax values. 738 stars uniformly distributed over the selected sky area (these are mostly Hipparcos stars) have $\varepsilon_{\pi}<6$ mas and $\pi>\varepsilon_{\pi}$ providing sufficiently accurate estimations of distances up to 500 pc (see Fig. 4) only for a few stars.

\subsection{Photometric data}

It is possible to divide the sources of the used data into several groups in accordance with the photometric accuracy, the magnitude ranges and the spatial distribution of photometric data.

From the point of photometric accuracy, photoelectric observations have the highest priority. There is a number

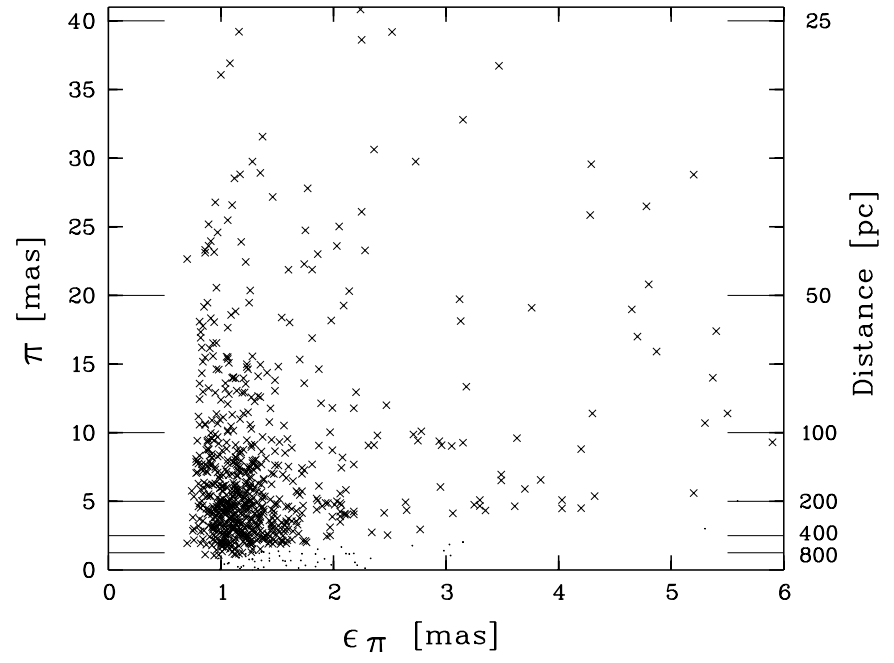

Fig. 4. Trigonometric parallaxes in the $\mathrm{CC}$ versus their rms errors (points). Stars with $\pi>\varepsilon_{\pi}$ are marked with crosses. The right vertical axis shows the distance scale corresponding to the parallax scale at the left.

of publications giving results of photoelectric multi-color photometry reaching $V_{\lim } \approx 13 \mathrm{mag}$ in a field covered by the shallow survey. Guetter (1977) gives results of original $U B V I J H K L$ photometry for 15 stars and absorption data for 111 stars. Seyfert et al. (1960) provide $U B V$ photoelectric photometry for 135 stars. Harris (1956) determined $U B V$ magnitudes and color-excesses for 13 stars. We also used the $V$ magnitudes and interstellar absorption values $A_{V}$ from Černis $(1990,1993)$ determined on the basis of observations in the Vilnius photometric system, where the $V$ magnitudes are very close to the Johnson $V$ system. All these data have errors less than $0.01 \ldots$ $0.02 \mathrm{mag}$ and were included in the $\mathrm{CC}$ with the highest priority.

For the remaining stars of the shallow survey, accurate and homogeneous $B_{\mathrm{T}}, V_{\mathrm{T}}$ magnitudes were provided by Tycho (their median errors are $0.01 \ldots 0.02 \mathrm{mag}$ for bright stars with $V<9$ mag and up to 0.06 mag for the rest). These data were transformed to the Johnson system. The intermediate correction was done with:

$V_{\mathrm{T}}^{\mathrm{c}}=V_{\mathrm{T}}-0.09(B-V)_{\mathrm{T}}+\delta_{V}$,

$B_{\mathrm{T}}^{\mathrm{c}}=B_{\mathrm{T}}-0.24(B-V)_{\mathrm{T}}+\delta_{(B-V)}$,

i.e. using recommendations from the Introduction to the Tycho catalogue (ESA 1997) and including additional terms $\delta_{V}$ and $\delta_{(B-V)}$. We determined these additional corrections using the ground-based photometric data in the Johnson system for these stars included in the Hipparcos/Tycho catalogues. The corrections depend nonlinearly on colour and can reach 0.02 and $0.04 \mathrm{mag}$, respectively. The corrected values underwent further reductions (as is shown below) to get a uniform photometric system over the CC.

Additionally, we used $V_{\mathrm{CMC}}$ magnitudes determined from observations of the automatic Carlsberg Meridian Circle with rms errors of $0.03 \ldots 0.1$ mag. If no other data 
Table 6. Coefficients of photometric transformation equations.

\begin{tabular}{lccc}
\hline $\begin{array}{l}\text { Magnitude, } \\
\text { catalogue }\end{array}$ & $\begin{array}{c}\text { rms error } \\
{[\mathrm{mag}]}\end{array}$ & $a$ & $\begin{array}{c}b \\
{[\mathrm{mag}]}\end{array}$ \\
\hline$B_{\mathrm{T}}^{\mathrm{c}}$, Tycho-2 & 0.10 & $-0.0090 \pm 0.0011$ & $+0.117 \pm 0.012$ \\
$V_{\mathrm{T}}^{\mathrm{c}}$, Tycho-2 & 0.09 & $-0.0042 \pm 0.0011$ & $+0.046 \pm 0.011$ \\
$B_{\mathrm{T}}^{\mathrm{c}}$, Tycho-1 & 0.021 & $-0.0006 \pm 0.0002$ & $+0.027 \pm 0.003$ \\
$V_{\mathrm{T}}^{\mathrm{c}}$, Tycho-1 & 0.003 & $+0.0000 \pm 0.0000$ & $-0.000 \pm 0.000$ \\
$m_{\mathrm{pg}}$, PPM-N & 0.25 & $-0.014 \pm 0.004$ & $+0.231 \pm 0.041$ \\
$V$, CMC11 & 0.024 & $-0.021 \pm 0.004$ & $+0.270 \pm 0.040$ \\
$B$, FONAC & 0.15 & $-0.015 \pm 0.002$ & $+0.195 \pm 0.020$ \\
$V$, FONAC & 0.17 & $-0.016 \pm 0.002$ & $+0.158 \pm 0.022$ \\
\hline
\end{tabular}

were available, we used $m_{\text {pg }}$ magnitudes from the PPM-N catalogue. Their accuracy is about $0.2 \mathrm{mag}$ (Kharchenko et al. 1995). If none of the above mentioned sources were available, we used photometric data given in SIMBAD.

All transformations to the Johnson system were carried out with

$m_{\mathrm{J}}-m=a m+b$,

where $m_{\mathrm{J}}$ is the magnitude in Johnson's system, $m$ is a magnitude from the data source, and $a, b$ are the transformation coefficients, derived from a least-squares method solution of the above equations for common stars with the rejection of stars not passing a 3 -sigma criterion. The results of the transformation are given in Table 6 .

Every star in the shallow survey got $B$ and/or $V$ magnitudes in the Johnson system and photometric errors copied from the corresponding catalogue or taken from Table 6.

In the field of our deep survey, photometric data were taken from the publications listed in Table 2. These catalogues were obtained by different detectors and methods: photographic, photoelectric, optical and infrared CCDs.

Strom et al. (1974) carried out photoelectric observations in the $U B V$ (19 stars) and $K$ (29 stars) bands. Their paper contains mostly the central part of IC 348 with stars from the list of Gingrich (1922). Lada \& Lada (1995) obtained deep near-infrared $J H K$ imaging data for 510 stars in an area of about 385 square arcmin using the Simultaneous Quad Infrared Imaging Device on the KPNO $1.3 \mathrm{~m}$ telescope with a resolution of 1.36 arcsec per pixel. Trullols \& Jordi (1997) carried out deep UBVRI Johnson-Cousins CCD photometry for 123 stars in an area of about 100 square arcmin at Calar Alto, Spain using the $2.2 \mathrm{~m}, 1.52 \mathrm{~m}$ and $1.23 \mathrm{~m}$ telescopes with pixel sizes of $0.28,0.33$ and 0.502 arcsec, respectively. Herbig (1998) determined deep $B V R I$ Johnson-Cousins stellar magnitudes for about 260 stars in an area of about 200 square arcmin with $24 \mu \mathrm{m}$ pixel size at the $\mathrm{f} / 10$ focus of the $2.2 \mathrm{~m}$ telescope.

The S99 catalogue contains deep $U B V R$ photographic magnitudes determined from POSS and Tautenburg Schmidt plates for which data from Trullols \& Jordi (1997) served as standards. The reduction methods and the achieved accuracy are described in detail in S99.
For common stars from various lists of the deep survey area, simple average magnitudes were computed and included in the $\mathrm{CC}$, whereas non overlapping photometric data were copied to the $\mathrm{CC}$ as they are.

\subsection{X-ray data}

Preibisch et al. (1996) detected 114 X-ray sources in deep pointings with the ROSAT Position Sensitive Proportional Counter (PSPC) and the High Resolution Imager (HRI). Observations were carried out in an area of about one square degree. PSPC count rates and their errors are included in the CC. Additionally, in the SIMBAD data base, we found 130 other X-ray sources distributed across the CC area. As mentioned in Sect. 3.1, the object identification was made on the basis of coordinate coincidence with a match radius shown in Table 4 . Since the shallow survey includes proper motions for stars down to $13 \ldots$ $14 \mathrm{mag}$, we were able to identify in the IC 348 neighbourhood two times more X-ray sources than in S99, where bright stars were missing or distorted due to crowding effects. Altogether, out of $244 \mathrm{X}$-ray sources included in the CC, 118 were identified with optical objects, and 110 of them have measured proper motions.

\subsection{Spectral classification data}

The Hipparcos, CMC11 and PPM-N catalogues contain MK and/or HD spectral classification. We also incorporated smaller lists of relevant spectral classes found in the literature. Černis $(1990,1993)$ provided MK classes estimated on the basis of the Vilnius photometric system. The MK classification for a number of stars was obtained by Seyfert et al. (1960) and Guetter (1977). Strom et al. (1974) carried out MK classification on the basis of spectrograms obtained with the Kitt Peak National Observatory (KPNO) image tube. Luhman et al. (1998) performed deep infrared and optical spectroscopy for 140 stars within the $5 \times 5$ arcmin core of IC 348 and published their spectral classes. Classification spectra of about 80 stars were obtained with the Multiple Object Spectrograph in the spectral region from about $5800 \AA$ to $7100 \AA$ by Herbig (1998). Table 7 lists all the data sources ordered by priority.

Altogether, 7018 stars both in the shallow and in the deep parts of the CC have a spectral classification. Additionally, we included the equivalent widths of $\mathrm{H}_{\alpha}$ emission for 124 stars, measured by Herbig (1998) and Luhman et al. (1998).

\subsection{Radial velocities}

The radial velocities are taken from SIMBAD and from the latest version of the Barbier-Brossat \& Figon (2000) catalogue of stellar radial velocities. There are 330 stars with these data. The distribution of these stars over the area is not homogeneous due to large selection effects. 
Table 7. Sources of spectral classification data.

\begin{tabular}{lc}
\hline Catalogue & Classification \\
\hline Černis (1990, 1993) & MK \\
Guetter (1977) & MK \\
Herbig (1998) & MK \\
Luhman et al. (1998) & MK \\
SIMBAD (Nov. 1999 version) & MK/HD \\
Hipparcos (Input catalogue) & MK/HD \\
PPM-N & HD \\
CMC11 & HD \\
Seyfert et al. (1960) & HD \\
\hline
\end{tabular}

\section{Stellar motions and Per OB2 membership}

In an area of one square degree around IC 348, S99 found two populations in addition to foreground field stars. With the extensive data described above it is worth investigating whether this holds also for the wider field covered by the CC.

Due to the high degree of completeness of the proper motion data (see Table 3), we consider the proper motions as the main tool for the segregation of the CC population. Nevertheless, the first step to reduce the contamination of the sample by field stars made use of the photometric data from the CC. In accordance with a preliminary $V-(B-V)$ colour-magnitude diagram (CMD), we excluded all stars with $V<10(B-V)$ from a further analysis. This group of about 6000 stars is clearly separated in the CMD from the bulk of the CC stars and is interpreted as field red giants, i.e. definitely non members of the association.

The Per OB2 association lies near the Galactic anticenter direction $\left(l, b \approx 161^{\circ},-15^{\circ}\right)$ and has a small peculiar tangential motion. Thus, proper motions in this area reflect mainly the differential Galactic rotation and the motion of the Sun with respect to the Local Standard of Rest (LSR). Therefore, the kinematic parameters of the association are close to average parameters of the field stars, and its proper motion distribution is considerably overlapping with that of the field stars so that additional measurements are necessary to segregate the field stars. Since the CC contains accurate trigonometric parallaxes for a number of stars, they can be used as an additional membership criterion for brighter stars.

In general, for a set of $K$ stellar parameters $t=$ $\left\{\mu_{X}, \mu_{Y}, \pi, V_{\mathrm{R}}, \ldots\right\}$ for each star $i$ one can define a membership probability as:

$P_{t}^{i}=\exp \left[-\frac{0.5}{K} \sum_{j=1}^{K}\left(\frac{t_{j}^{i}-\bar{t}_{j}}{\varepsilon_{t_{j}}^{i}}\right)^{2}\right] \times 100 \%$,

where $t_{j}^{i}$ and $\varepsilon_{t_{j}}^{i}$ are a value and mean error of the $j$ th parameter for the $i$ th star, and $\bar{t}_{j}$ is an average of the $j$ th parameter over a sample. This means that for each star, one can compute various kinds of probability, depending of the number of parameters involved: e.g. the propermotion based probability $\left(P_{\mu}\right)$ or proper-motion-parallax
Table 8. Average motions and parallaxes of subsamples of $A$ and $C$ populations.

\begin{tabular}{ccccc}
\hline Sample & $\begin{array}{c}\text { No of } \\
\text { stars }\end{array}$ & $\begin{array}{c}\bar{\mu}_{X} \\
{[\mathrm{mas} / \mathrm{yr}]}\end{array}$ & $\begin{array}{c}\bar{\mu}_{Y} \\
{[\mathrm{mas} / \mathrm{yr}]}\end{array}$ & $\begin{array}{c}\bar{\pi} \\
{[\mathrm{mas}]}\end{array}$ \\
\hline$A_{\mu}$ & 75 & $+3.09 \pm 0.12$ & $-4.25 \pm 0.14$ & - \\
$C_{\mu}$ & 58 & $+4.84 \pm 0.15$ & $-7.36 \pm 0.15$ & - \\
$A_{\mu, \pi}$ & 10 & $+3.67 \pm 0.44$ & $-5.45 \pm 0.51$ & $+3.77 \pm 0.18$ \\
$C_{\mu, \pi}$ & 8 & $+5.14 \pm 0.64$ & $-8.31 \pm 0.54$ & $+4.76 \pm 0.16$ \\
\hline
\end{tabular}

$\left(P_{\mu, \pi}\right)$ membership probability and so on. If the probability exceeds $61 \%$ (the deviation from the average is less than $\varepsilon_{t_{j}}^{i}$ ), we regard such a star as "probable" member of an ensemble. A star with the probability less than $1 \%$ (the deviation is greater than $3 \varepsilon_{t_{j}}^{i}$ ) is regarded as definite non member of the sample. In intermediate cases (probability between $61 \%$ and $14 \%$ or between $14 \%$ and $1 \%$ ) the star is considered to be possible sample member or possible non-member, respectively.

In order to check the presence of the two populations found by $\mathrm{S} 99$, we selected stars brighter than $V=11.6$ (i.e., down to the completeness limit of the $\mathrm{CC}$ ) which are distributed over the complete CC area. Further, we restricted our sample to stars with the most accurate proper motions in our data set $\left(\varepsilon_{\mu} \leq 2 \mathrm{mas} / \mathrm{yr}\right)$. According to the method described in Kharchenko \& Schilbach (1995), we analysed the two-dimensional distribution functions of the proper motions in the studied field and derived the initial parameters of the distributions. Then the parameters where improved iteratively, and as a result, we selected 133 most probable members of these populations. The analysis of this sample confirms the existence of two distinct, although very close in proper motion space stellar populations, which we call populations $A_{\mu}$ and $C_{\mu}$. Their average proper motions are given in Table 8 .

Since both the $A_{\mu}$ and $C_{\mu}$ populations are distributed over a significant part of the area studied, we considered them rather as a property of a larger structure such as the association itself than of a particular cluster, as S99 did. The working assumption was then that both populations represent association members which are located at different distances from the Sun. This assumption can be considered as reasonable since the distance to the association seems to be comparable to its depth.

To prove this assumption, we selected the stars with the most accurate parallax determinations $\left(\varepsilon_{\pi} \leq 2\right.$ mas, $\left.\pi / \varepsilon_{\pi}>3\right)$ available in the samples. These stars were used to compute average motions and parallaxes of the samples $A_{\mu, \pi}$ and $C_{\mu, \pi}$ shown in Table 8 . We can conclude that the mean proper motions are practically identical with those of the samples $A_{\mu}$ and $C_{\mu}$. The parallaxes from Table 8 indicate that population $A$ is in average more distant than population $C$.

Figure 5 shows the distribution of probable $\left(P_{\mu, \pi}>\right.$ $61 \%)$ members of $A_{\mu, \pi}$ and $C_{\mu, \pi}$ samples in vector diagrams and in parallax - velocity planes. Since for these stars we are able to compute distances, we can evaluate 

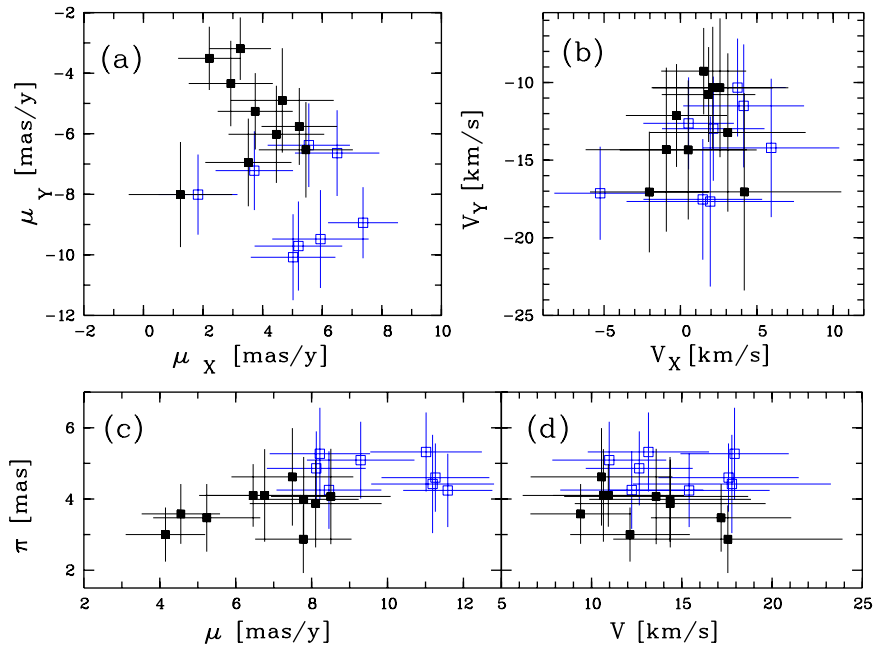

Fig. 5. Relation between kinematics and parallaxes of stars of $A_{\mu, \pi}$ (filled squares) and $C_{\mu, \pi}$ (open squares) samples. These samples consist of probable members $\left(P_{\mu, \pi}>61 \%\right)$ with the most accurate parallax measurements available in this area $\left(\varepsilon_{\pi} \leq 2\right.$ mas, $\left.\pi / \varepsilon_{\pi}>3\right)$. Panels $\left.\mathbf{a}\right)$ and $\left.\mathbf{b}\right)$ proper motion and tangential velocity vector diagrams; panels c) and $\mathbf{d}$ ) proper motions and tangential velocities versus parallaxes. The bars indicate the standard errors.

reasonably accurate tangential velocities for them which should, in contrast to the proper motions, not depend on the distance. We compute tangential velocities with

$V_{X, Y}=\frac{4.74 \cdot \mu_{X, Y}}{\pi}$,

and correct them for the Solar motion with respect to the LSR, and for differential rotation of the Galaxy. The Solar motion in the Galactic coordinate system was taken according to Dehnen \& Binney (1998) as $(U, V, W)=$ $(-10.0 \pm 0.4,+5.2 \pm 0.6,+7.2 \pm 0.4) \mathrm{kms}^{-1}$. Values of Oort constants were taken from Feast \& Whitelock (1997): $A=+14.82 \pm 0.84$, and $B=-12.37 \pm 0.64 \mathrm{~km} \mathrm{~s}^{-1} \mathrm{kpc}$.

From the comparison of the proper motion and tangential velocity vector diagrams (panels a and b of Fig. 5), we conclude that the population segregation which is evident in panel a is much less obvious in panel b. This implicitly supports our assumption. The data in panels $\mathrm{c}$ and $\mathrm{d}$, where proper motion and tangential velocities are confronted with parallaxes, directly confirm our idea. Both panels indicate a separation of the populations with their distance. Whereas proper motions show a straight correlation with parallaxes, the tangential velocities do not indicate any trend.

In Fig. 6 we show the average proper motion vector diagram of the samples from Table 8, as well as the S99 mean proper motions of their "cluster" and "distant field stars" groups corrected by Eqs. (1). From Fig. 6 we conclude that the centers of the proper motion distributions derived for the $A$ and $C$ samples coincide within their errors with the results obtained in S99 for "distant field" and "cluster" stars, although the S99 data cover only the one square degree field around the IC 348 cluster.

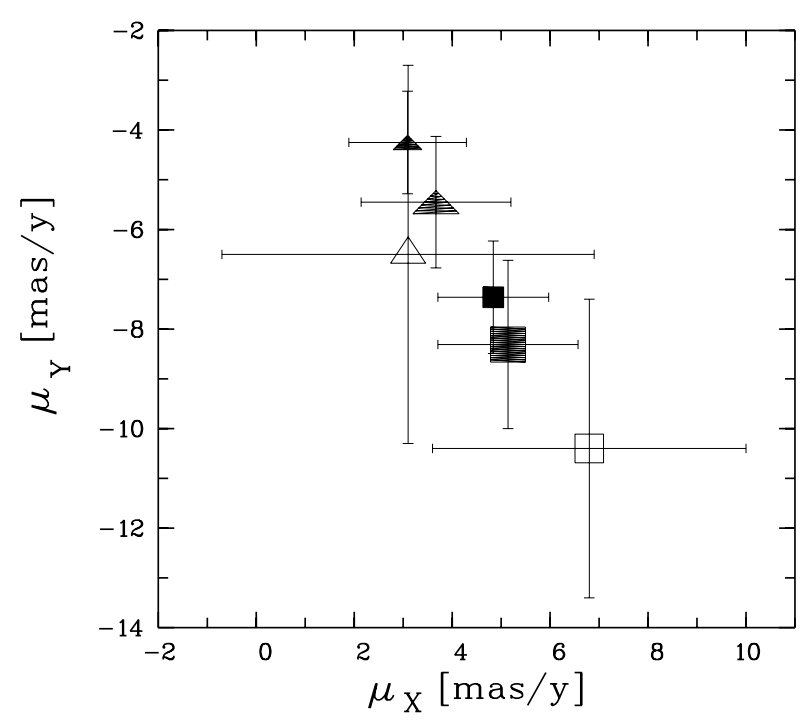

Fig. 6. Average proper motions of the considered samples. Filled triangles correspond to $A_{\mu}$ (small) and $A_{\mu, \pi}$ (large) samples, filled squares correspond to $C_{\mu}$ (small) and $C_{\mu, \pi}$ (large) samples. Open symbols show the average proper motions of "distant field" (triangle) and "cluster" (square) star groups of S99. The error bars denote the corresponding standard deviations.

Though both populations seem to be discernible by their average distances, they are adjacent to each other, and can be regarded as parts of a single structure. Note, that in the face-on plane both groups are mutually penetrating, i.e. stars of $A$ and $C$ populations occupy the same area (as it was mentioned by Herbig 1998 and by S99 for the case of IC 348). We conclude that the parameters in Table 8 give a reasonable model of the spatial structure of the Per OB2 star-forming region, in which IC 348 occupies its front layer at the distance of about $210 \mathrm{pc}$, whereas the association itself is displaced along the line of sight to a distance of 265 pc. Due to the Lutz-Kelker bias, the derived values must be considered as lower limits of the distance estimates rather than the mean distances of IC 348 and Per OB2.

Our analysis shows that, in order to get a meaningful estimate of the membership probabilities for the Per OB2 association, one has to rely on tangential velocities which require both the knowledge of proper motions and of distances. For the majority of the CC stars, sufficiently accurate distance estimates are not yet available. Nevertheless, as a first approximation, the membership evaluation can be carried out from the proper motions alone. For each star, the membership probability $P_{\mu}$ can be computed from the proper motion and its mean errors for a star in the CC by use of Eq. (2) and data in Table 8. In this case, we should keep in mind that both $A$ and $C$ populations belong to the association.

Out of about 30000 stars occupying an area of about 320 square degrees, we found $1014+1225$ probable members based on proper motion probabilities $\left(P_{\mu}>61 \%\right)$, respectively of the $A+C$ population. Down to the 
completeness limit of the CC $(V=11.6)$, which is one of the most important parameters in the study of general structure and kinematics of the Per OB2 association, the corresponding numbers are $794+559$. The comparison of our membership probabilities with those, derived by de Zeeuw et al. (1999) from the Hipparcos data, shows that both approaches are in reasonable agreement: out of 39 Per OB2 members detected by de Zeeuw et al. (1999) in the area covered by the CC, we classified 24 stars as probable members and 14 stars as possible members, whereas only one star was rejected as a field star.

\section{Summary}

In this paper we describe the construction of a Compiled Catalogue for a nearby region of star formation with the Per OB2 association and the young cluster IC 348 .

We describe the reductions of various parameters which enable us to put the data into a single system regarded as a de facto present-day standard: the coordinates and proper motions are in the Hipparcos system, whereas the photometry is in the Johnson system. Typical accuracies of the CC stars with $V<12$ mag are: $1 \ldots 20$ mas for the coordinates, $1 \ldots 3 \mathrm{mas} / \mathrm{yr}$ for the proper motions and $0.01 \ldots 0.05$ mag for the $B, V$ magnitudes. For about 7000 of $30000 \mathrm{CC}$ stars, we collected parallaxes and spectral classes needed for a comprehensive study of the morphology and history of star formation in the region.

From the analysis of the proper motion data, the stellar population of the complex can be split into two groups distributed over the whole region studied. In the face-on plane, the members of both groups occupy the same area but they differ by their average distance from the Sun. We interpret this result as evidence of a considerable depth of the Per OB2 complex. Down to the completeness limit of the Compiled Catalogue, we have selected more than 1000 probable members of the Per OB2 complex, mainly based on the proper motion criterion.

The complete data from the Compiled Catalogue provide a basis for the study of the star formation process in the Per OB2 association from the analysis of the structure and contents of stellar population in this region. The next paper in this series deals with structure and kinematics of Per OB2.

The electronic version of the catalogue is available from the Centre des Données astronomiques de Strasbourg (CDS), France via anonymous ftp from cdsarc.u-strasbg.fr (130.79.128.5) or via http://cdsweb.u-strasbg.fr/cgi-bin/qcat?J/A+A/384/145

Acknowledgements. Part of this work was supported by the Deutsche Forschungsgemeinschaft, DFG project number $113 / 380 / \mathrm{O}(\mathrm{R})$. RDS gratefully acknowledges financial support from the Deutsches Zentrum für Luft- und Raumfahrt (DLR) (Förderkennzeichen 50 OI 0001). We also acknowledge the use of the SIMBAD database operated at the CDS. We thank the referee, Dr. F. Ochsenbein for useful comments which helped us to improve the paper.

\section{References}

Ambarzumian, V. A. 1947, in Stellar Evolution and Astrophysics, Armenian. Acad. Sci.

Ambarzumian, V. A. 1949, Dokl. Acad. Nauk SSSR, 68, 22

Barbier-Brossat, M., \& Figon, P. 2000, A\&AS, 142, 217

Belikov, A. N., Kharchenko, N. V., Piskunov, A. E., \& Schilbach, E. 1999, A\&AS, 134, 525

Belikov, A. N., Kharchenko, N. V., Piskunov, A. E., \& Schilbach, E. 2000a, A\&A, 358, 886

Blaauw, A. 1952, BAN, 11, 405

Blaauw, A. 1964, ARA\&A, 2, 213

Carlsberg Meridian Catalogue La Palma, Numbers 1 to 11, Observations of positions of stars and planets: May 1984 to May 1998, 1999, Copenhagen University Observatory, Royal Greenwich Observatory and Real Instituto y Observatorio de la Armada en San Fernando, CD-ROM distribution

Černis, K. 1990, ApSS, 166, 315

Černis, K. 1993, Baltic Astr., 2, 214

Dehnen, W., \& Binney, J. J. 1998, MNRAS, 298, 387

ESA 1997, The Hipparcos and Tycho Catalogues, SP-1200

Feast, M. W., \& Whitelock, P. 1997, MNRAS, 291, 683

Fredrick, L. W. 1956, AJ, 61, 437

Gingrich, C. H. 1922, ApJ, 56, 139

Guetter, H. H. 1977, AJ, 82, 598

Harris III, D. L. 1956, ApJ, 123, 371

Herbig, G. H. 1998, ApJ, 497, 736

Høg, E., Kuzmin, A., Bastian, U., et al. 1998, A\&A, 335, L65

Høg, E., Fabricius, C., Makarov, V. V., et al. 2000, Astrometric and photometric reference catalogue of the 2.5 million brightest stars covering the entire sky, Copenhagen, CDROM distribution

Høg, E., Fabricius, C., Makarov, V. V., et al. 2000, A\&A, 357, 367

Kislyuk, V., Yatsenko, A., Ivanov G., Pakulyak L., \& Sergeeva T. 1999, in Motion of Celestial Bodies, Astrometry and Astronomical Reference Frames, Journées 1999 \& IX Lohrman Kolloquim, Dresden, Germany, 1315 September 1999

Kharchenko, N., \& Schilbach, E. 1995, Astron. Nachr., 316, 91 Kharchenko, N., Molotaj, O., Pakulyak, L., \& Tel'nyukAdamchuk, V. 1995, Astron. Nachr., 316, 401

Lada, E. A., \& Lada, C. J. 1995, AJ, 109, 1682

Luhman, K. L., Rieke, G. R., Lada, C. J., \& Lada, E. A. 1998, ApJ, 508, 347

Preibisch, T., Zinnecker, H., \& Herbig, G. H. 1996, A\&A, 310, 456

Röser, S., \& Bastian, U. 1991, PPM Star Catalogue 1, 2 (Spectrum Academischer Verlag, Heidelberg, Berlin, New York)

Scholz, R.-D., Brunzendorf, J., Ivanov, G., et al. 1999, A\&AS, 137, 305 (S99)

Seyfert, C. K., Hardie, R. H., \& Grenchik, R. T. 1960, ApJ, 132,58

Strom, S. E., Strom, K. M., \& Carrasco, L. 1974, PASP, 86, 798

Trullols, E., \& Jordi, C. 1997, A\&A, 324, 549

Urban, S. E., Corbin, T. E., Wycoff, G. L., et al. 1998a, AJ, 115,1212

Urban, S. E., Corbin, T. E., \& Wycoff, G. L. 1998b, AJ, 115, 2161

de Zeeuw, P. T., Hoogerwerf, R., de Bruijne, J. H. J., Brown, A. G. A., \& Blaauw, A. 1999, AJ, 117, 354 